\title{
On existence-uniqueness results for proportional fractional differential equations and incomplete gamma functions
}

\author{
Zaid Laadjal' ${ }^{1}$, Thabet Abdeljawad $2,3,4^{*}$ (D) and Fahd Jarad ${ }^{5}$
}

\section{"Correspondence:}

tabdeljawad@psu.edu.sa

${ }^{2}$ Department of Mathematics and

General Sciences, Prince Sultan

University, P.O. Box 66833, 11586

Riyadh, Saudi Arabia

${ }^{3}$ Department of Medical Research,

China Medical University, 40402

Taichung, Taiwan

Full list of author information is

available at the end of the article

\begin{abstract}
In this article, we employ the lower regularized incomplete gamma functions to demonstrate the existence and uniqueness of solutions for fractional differential equations involving nonlocal fractional derivatives (GPF derivatives) generated by proportional derivatives of the form$$
D^{\rho}=(1-\rho)+\rho D, \quad \rho \in[0,1],
$$

where $D$ is the ordinary differential operator.

Keywords: Incomplete gamma function; Generalized proportional fractional differential equation; Existence; Uniqueness
\end{abstract}

\section{Introduction}

Over the last decades there has been an extensive use of fractional dynamic equations in modeling and describing complex and chaotic systems [1-6]. This fact has motivated researchers to discover new fractional operators. After Riemann-Liouville fractional derivatives, Caputo fractional derivatives were defined to transform constant functions to zero and hence have similar properties as ordinary derivatives. On the other hand, some researchers have spent their efforts to define more general classes of fractional operators by inserting more general or new kernels. Since the appearance of the concept of conformable derivatives, which allow the derivation up to arbitrary order and resemble ordinary derivatives, in [7] and their modifications in [8, 9], several researchers realized that conformable type derivatives can be used to produce nonlocal more generalized fractional derivatives $[10,11]$. Indeed, in [10], the authors used the conformable derivatives presented in [8] to present a class of generalized nonlocal fractional derivatives, called conformable fractional derivatives, slightly different from the so-called Katugampola [12, 13]. In fact, the derivatives in [10] and the Katugampola are characterized as fractional derivatives of a function with respect to another function $g(t)$, with $g(t)=\frac{t^{\rho}}{\rho}$ and $g(t)=\frac{(t-a)^{\rho}}{\rho}$, respectively [14]. Also, the authors in [11] used particular versions of the proportional derivatives presented in [9], called modified conformable derivatives, to present the fractional counterpart pro-

(c) The Author(s) 2020. This article is licensed under a Creative Commons Attribution 4.0 International License, which permits use sharing, adaptation, distribution and reproduction in any medium or format, as long as you give appropriate credit to the original author(s) and the source, provide a link to the Creative Commons licence, and indicate if changes were made. The images or other third party material in this article are included in the article's Creative Commons licence, unless indicated otherwise in a credit line to the material. If material is not included in the article's Creative Commons licence and your intended use is not permitted by statutory regulation or exceeds the permitted use, you will need to obtain permission directly from the copyright holder. To view a copy of this licence, visit http://creativecommons.org/licenses/by/4.0/. 
portional derivatives and integrals. Later, the authors in $[15,16]$ generalized proportional derivatives and used them to generate more generalized classes of nonlocal fractional integrals and derivatives, and in [17] the authors discussed a new type of fractional operators combining proportional and classical derivatives/integrals. Besides, there have been many attempts to generate fractional operators with more complicated kernels with the hope to describe complex systems more accurately. Some authors thought of replacing the singular kernels with power law by nonsingular kernels with either exponential law [18] or MittagLeffler law [19] via ML kernel and via generalized ML kernel. For the interest of readers, we attract their attention to the recent work where the author studied the relationships between the model of Prabhakar depending on fractional integrals with generalized ML kernels and Atangana-Baleanu model in [19] and its extension in [20].

Fixed point techniques are always used to prove existence and uniqueness of ordinary and fractional dynamic equations [21-27]. It turns out that the structure of the kernel of fractional operator affects the applied analysis technique in proving the existence and uniqueness of solution or its stability criteria due to the natural appearance of the exponential function in the kernel of proportional fractional point technique in proving the existence and uniqueness of solutions for fractional differential equations in the setting of GPF derivatives. Indeed, we investigate the following Cauchy problem:

$$
\left\{\begin{array}{l}
{ }^{c} D_{a}^{\alpha, \rho} u(t)=f(t, u(t)), \quad a<t<b, \alpha>0, \\
u^{(k)}(a)=b_{k}, \quad k=0,1, \ldots, n-1,
\end{array}\right.
$$

where $\rho \in(0,1], n=-[-\alpha],-\infty<a<b<+\infty, b_{k} \in \mathbb{R}$, and ${ }^{c} D_{a}^{\alpha, \rho}$ denotes the Caputo proportional fractional derivative of order $\alpha, f:[a, b] \times \mathbb{R} \rightarrow \mathbb{R}$ is a given continuous nonlinear function.

Further, we will also obtain a result for the following fractional differential equation involving proportional fractional order with initial conditions:

$$
\left\{\begin{array}{l}
{ }^{R} D_{a}^{\alpha, \rho} u(t)=f(t, u(t)), \quad a<t<b, \alpha>0, \\
{ }^{R} D_{a}^{\alpha-k, \rho} u(a)=b_{k}, \quad k=1, \ldots, n,
\end{array}\right.
$$

where $n=-[-\alpha],{ }^{R} D_{a}^{\alpha, \rho}$ denotes the generalized proportional integral of RiemannLiouville type of order $\alpha$.

The paper is organized as follows: Sect. 2 presents some definitions and results needed in the rest of the article. Sect. 3 discusses new lemmas needed for the proofs of the existence and uniqueness of the Cauchy problems proposed in Sect. 4.

\section{Preliminaries}

Definition 2.1 ([5]) Let $\alpha \geq 0$. The left fractional integral of Riemann-Liouville type of the function $\Psi$ is defined by $\left(I_{a}^{0} \Psi\right)(t)=\Psi(t)$ and

$$
\left(I_{a}^{\alpha} \Psi\right)(t)=\frac{1}{\Gamma(\alpha)} \int_{a}^{t}(t-\tau)^{\alpha-1} \Psi(\tau) d \tau \quad \text { for } \alpha>0
$$

where $t \in[a, b]$. 
Definition 2.2 ([5]) Let $\alpha \geq 0$. The left fractional derivative of Caputo type of the function $\Psi \in C^{(n)}[a, b]$ is defined by ${ }^{c} D_{a}^{0} \Psi(t)=\Psi(t)$ and

$$
\begin{aligned}
{ }^{c} D_{a}^{\alpha} \Psi(t) & =J_{a}^{n-\alpha} \Psi^{(n)}(t) \\
& =\frac{1}{\Gamma(n-\alpha)} \int_{a}^{t}(t-\tau)^{n-\alpha-1} \Psi^{(n)}(\tau) d \tau \quad \text { for } \alpha>0,
\end{aligned}
$$

where $n-1<\alpha \leq n, n \in \mathbb{N}$.

Definition 2.3 ([11]) Let $\rho \in(0,1]$ and $\alpha \geq 0$. The left generalized proportional integral of Riemann-Liouville type of the function $\Psi \in L^{1}[a, b]$ is defined by $\left(J_{a}^{0, \rho} \Psi\right)(t)=\Psi(t)$ and

$$
\left(J_{a}^{\alpha, \rho} \Psi\right)(t)=\frac{1}{\rho^{\alpha} \Gamma(\alpha)} \int_{a}^{t}(t-\tau)^{\alpha-1} e^{\frac{\rho-1}{\rho}(t-\tau)} \Psi(\tau) d \tau \quad \text { for } \alpha>0
$$

where $t \in[a, b]$.

Definition 2.4 ([11]) Let $\rho \in(0,1]$ and $\alpha \geq 0$. The left generalized proportional derivative of Caputo type of the function $\Psi \in C^{(n)}[a, b]$ is defined by ${ }^{c} D_{a}^{0, \rho} \Psi(t)=\Psi(t)$ and

$$
\begin{aligned}
{ }^{c} D_{a}^{\alpha, \rho} \Psi(t) & =J_{a}^{n-\alpha, \rho}\left(D^{n, \rho} \Psi\right)(t) \\
& =\frac{1}{\rho^{\alpha} \Gamma(n-\alpha)} \int_{a}^{t}(t-\tau)^{n-\alpha-1} e^{\frac{\rho-1}{\rho}(t-\tau)}\left(D^{n, \rho} \Psi\right)(\tau) d \tau \quad \text { for } \alpha>0,
\end{aligned}
$$

where $n-1<\alpha \leq n, n \in \mathbb{N}$, and $\left(D^{1, \rho} \Psi\right)(t)=\left(D^{\rho} \Psi\right)(t)=(1-\rho) \Psi(t)+\rho \Psi^{\prime}(t)$, and

$$
\left(D^{n, \rho} \Psi\right)(t)= \begin{cases}\Psi(t), & \text { for } n=0, \\ \left(D^{n, \rho} \Psi\right)(t)=(\underbrace{D^{\rho} D^{\rho} \cdots D^{\rho}}_{n \text { times }} \Psi)(t) & \text { for } n \geq 1 .\end{cases}
$$

Definition 2.5 ([11]) Let $\rho \in(0,1]$ and $\alpha \geq 0$. The left generalized proportional derivative of Riemann-Liouville type of the function $\Psi$ is defined by ${ }^{R} D_{a}^{0, \rho} \Psi(t)=\Psi(t)$ and

$$
\begin{aligned}
{ }^{R} D_{a}^{\alpha, \rho} \Psi(t) & =D^{n, \rho} J_{a}^{n-\alpha, \rho} \Psi(t) \\
& =\frac{D^{n, \rho}}{\rho^{n-\alpha} \Gamma(n-\alpha)} \int_{a}^{t}(t-\tau)^{n-\alpha-1} e^{\frac{\rho-1}{\rho}(t-\tau)} \Psi(\tau) d \tau \quad \text { for } \alpha>0,
\end{aligned}
$$

where $n-1<\alpha \leq n, n \in \mathbb{N}$.

Remark 2.6 Note that, for $\rho=1$, Definitions 2.3 and 2.4 reduce to the usual definitions of Riemann-Liouville fractional integral and Caputo fractional derivative, respectively.

Lemma 2.7 Let $n \in \mathbb{N}$ and $\Psi \in C^{(n)}[a, b]$. Then,

$$
\left(D^{n, \rho} \Psi\right)(t)=\rho^{n} \Psi^{(n)}(t)+\sum_{k=0}^{n-1} C_{n}^{k} \rho^{k}(1-\rho)^{n-k} \Psi^{(k)}(t) \quad \text { for } \rho \in(0,1]
$$

where $C_{n}^{k}=\left(\begin{array}{l}n \\ k\end{array}\right)=\frac{n !}{k !(n-k) !}$. 
Proof The proof follows by writing $D^{\rho}=(1-\rho)+\rho D$ and applying the binomial theorem.

Remark 2.8 Let $\rho \in(0,1]$ and $\alpha>0$ with $n-1<\alpha \leq n, n \in \mathbb{N}$. By using Lemma 2.7, we can write the definition of the left generalized proportional derivative of Caputo type of the function $\Psi \in C^{(n)}[a, b]$ as follows:

$$
{ }^{c} D_{a}^{\alpha, \rho} \Psi(t)=\rho^{n} J_{a}^{n-\alpha, 1} \Psi^{(n)}(t)+\sum_{k=0}^{n-1} C_{n}^{k} \rho^{k}(1-\rho)^{n-k} J_{a}^{n-\alpha, \rho} \Psi^{(k)}(t) .
$$

Proposition 2.9 ([11]) Let $\rho \in(0,1], \beta>0$, and $\alpha>0$ with $n-1<\alpha \leq n$ and $\Psi \in L^{1}[a, b]$, we have the following properties:

$$
\begin{aligned}
& \left(J_{a}^{\alpha, \rho}(x-a)^{\beta-1} e^{\frac{\rho-1}{\rho} x}\right)(t)=\frac{\Gamma(\beta)}{\rho^{\alpha} \Gamma(\alpha+\beta)}(t-a)^{\alpha+\beta-1} e^{\frac{\rho-1}{\rho} t} ; \\
& \left({ }^{R} D_{a}^{\alpha, \rho}(x-a)^{\beta-1} e^{\frac{\rho-1}{\rho} x}\right)(t)=\frac{\rho^{\alpha} \Gamma(\beta)}{\Gamma(\beta-\alpha)}(t-a)^{\beta-\alpha-1} e^{\frac{\rho-1}{\rho} t} ; \\
& \left(J_{a}^{\alpha, \rho} J_{a}^{\beta, \rho} \Psi\right)(t)=\left(J_{a}^{\beta, \rho} J_{a}^{\alpha, \rho} \Psi\right)(t)=\left(J_{a}^{\alpha+\beta, \rho} \Psi\right)(t) ; \\
& \left({ }^{c} D_{a}^{\alpha, \rho} J_{a}^{\alpha, \rho} \Psi\right)(t)=\Psi(t) ; \\
& \left({ }^{R} D_{a}^{\alpha, \rho} J_{a}^{\alpha, \rho} \Psi\right)(t)=\Psi(t) ; \\
& \left(J_{a}^{\alpha, \rho c} D_{a}^{\alpha, \rho} \Psi\right)(t)=\Psi(t)-\sum_{k=0}^{n-1} c_{k}(t-a)^{k} e^{\frac{\rho-1}{\rho}(t-a)}, \quad \Psi \in C^{(n)}[a, b],
\end{aligned}
$$

where $c_{k}=\frac{\left(D^{k, \rho} \Psi\right)(a)}{\rho^{k} k !}$

$$
\left(J_{a}^{\alpha, \rho R} D_{a}^{\alpha, \rho} \Psi\right)(t)=\Psi(t)-\sum_{k=1}^{n} q_{k}(t-a)^{\alpha-k} e^{\frac{\rho-1}{\rho}(t-a)},
$$

where $q_{k}=\frac{\left(J_{a}^{k-\alpha, \rho} \Psi\right)(a)}{\rho^{\alpha-k} \Gamma(\alpha-k+1)}$.

Definition 2.10 ([28, 29]) Let $\alpha \in \mathbb{C}(\Re(\alpha)>0)$, we have the following definitions:

The upper incomplete gamma function is defined by

$$
\Gamma(\alpha, t)=\int_{t}^{+\infty} y^{\alpha-1} e^{-y} d y
$$

The lower incomplete gamma function is defined by

$$
\gamma(\alpha, t)=\int_{0}^{t} y^{\alpha-1} e^{-y} d y
$$

The upper regularized incomplete gamma function is defined by

$$
Q(\alpha, t)=\frac{\Gamma(\alpha, t)}{\Gamma(\alpha)}
$$


The lower regularized incomplete gamma function is defined by

$$
P(\alpha, t)=1-Q(\alpha, t)=\frac{\gamma(\alpha, t)}{\Gamma(\alpha)} .
$$

The functions $P$ and $Q$ are also called "Incomplete gamma function ratios".

Lemma 2.11 ([28]) Let $\alpha \geq 0$. For all $t \geq 0$, we have the following properties:

$$
\begin{aligned}
& \Gamma(\alpha+1, t)=\alpha \Gamma(\alpha, t)+t^{\alpha} e^{-t}, \\
& \gamma(\alpha, t)=\Gamma(\alpha)-\Gamma(\alpha, t), \\
& \gamma(\alpha+1, t)=\alpha \gamma(\alpha, t)-t^{\alpha} e^{-t}, \\
& \int_{t_{1}}^{t_{2}} y^{\alpha-1} e^{-y} d y=\gamma\left(\alpha, t_{2}\right)-\gamma\left(\alpha, t_{1}\right), \quad t_{2} \geq t_{1}>0 .
\end{aligned}
$$

Lemma 2.12 Let $\alpha, \eta \in \mathbb{R}^{+}$. It is clear that $P(\alpha, \eta(t-a))$ is a nondecreasing function with respect to $t \in[a, b]$. Moreover,

$$
\begin{aligned}
& P(\alpha, \eta(t-a)) \in[0,1] \quad \text { or all } t \geq a, \\
& \max _{t \in[a, b]} P(\alpha, \eta(t-a))=\left.P(\alpha, \eta(t-a))\right|_{t=b}=P(\alpha, \eta(b-a)), \\
& \min _{t \in[a, b]} P(\alpha, \eta(t-a))=\left.P(\alpha, \eta(t-a))\right|_{t=a}=0 .
\end{aligned}
$$

\section{Incomplete gamma functions vs fractional proportional integrals}

In this section, we present new essential lemmas, which allow us to proceed in proving our main results about the existence and uniqueness of solutions for GPF differential equations.

Lemma 3.1 Let $\rho \in(0,1], \alpha>0$, and $f(t)=1$ for all $t \in[a, b]$. Then

$$
\left(J_{a}^{\alpha, \rho} 1\right)(t)= \begin{cases}\frac{P\left(\alpha, \frac{1-\rho}{\rho}(t-a)\right)}{(1-\rho)^{\alpha}}, & \text { for } \rho \in(0,1), \\ \left(I_{a}^{\alpha} 1\right)(t)=\frac{(t-a)^{\alpha}}{\Gamma(\alpha+1)}, & \text { for } \rho=1\end{cases}
$$

where the function P is defined by (22). Moreover,

$$
\lim _{\rho \rightarrow 1} \frac{P\left(\alpha, \frac{1-\rho}{\rho}(t-a)\right)}{(1-\rho)^{\alpha}}=\left(I_{a}^{\alpha} 1\right)(t)=\frac{(t-a)^{\alpha}}{\Gamma(\alpha+1)}
$$

and

$$
\max _{t \in[a, b]}\left(\lim _{\rho \rightarrow 1} \frac{P\left(\alpha, \frac{1-\rho}{\rho}(t-a)\right)}{(1-\rho)^{\alpha}}\right)=\frac{(b-a)^{\alpha}}{\Gamma(\alpha+1)} .
$$

Proof For $\rho \in(0,1)$, from Definition 2.3, we have

$$
\left(J_{a}^{\alpha, \rho} 1\right)(t)=\frac{1}{\rho^{\alpha} \Gamma(\alpha)} \int_{a}^{t}(t-\tau)^{\alpha-1} e^{\frac{\rho-1}{\rho}(t-\tau)} d \tau .
$$


Let $y=\frac{1-\rho}{\rho}(t-\tau)$, then $d y=-\frac{1-\rho}{\rho} d \tau$, or $d \tau=-\frac{\rho}{1-\rho} d y$. Hence, we have

$$
\begin{aligned}
\left(J_{a}^{\alpha, \rho} 1\right)(t) & =\frac{-1}{\rho^{\alpha} \Gamma(\alpha)} \int_{\frac{1-\rho}{\rho}(t-a)}^{0}\left(\frac{\rho}{1-\rho} y\right)^{\alpha-1} e^{-y} \frac{\rho}{1-\rho} d y \\
& =\frac{1}{(1-\rho)^{\alpha} \Gamma(\alpha)} \int_{0}^{\frac{1-\rho}{\rho}(t-a)} y^{\alpha-1} e^{-y} d y \\
& =\frac{\gamma\left(\alpha, \frac{1-\rho}{\rho}(t-a)\right)}{(1-\rho)^{\alpha} \Gamma(\alpha)} \\
& =\frac{P\left(\alpha, \frac{1-\rho}{\rho}(t-a)\right)}{(1-\rho)^{\alpha}} .
\end{aligned}
$$

Concerning the limit formula (31), we have

$$
\begin{aligned}
\lim _{\rho \rightarrow 1}\left(\frac{P\left(\alpha, \frac{1-\rho}{\rho}(t-a)\right)}{(1-\rho)^{\alpha}}\right) & =\lim _{\rho \rightarrow 1} \frac{1}{\rho^{\alpha} \Gamma(\alpha)} \int_{a}^{t}(t-\tau)^{\alpha-1} e^{\frac{\rho-1}{\rho}(t-\tau)} d \tau \\
& =\frac{1}{\Gamma(\alpha)} \int_{a}^{t}(t-\tau)^{\alpha-1} d \tau \\
& =\frac{(t-a)^{\alpha}}{\Gamma(\alpha+1)} .
\end{aligned}
$$

Finally, formula (32) is immediate, and hence the proof is completed.

Lemma 3.2 Let $X=C([a, b], \mathbb{R})$ be the Banach space of all continuous functions from $[a, b]$ to $\mathbb{R}$ endowed with the norm $\|u\|=\sup _{t \in[a, b]}|u(t)|$, and let $\rho \in(0,1], \alpha>0$, and $f \in X$. Then

$$
\left|\left(J_{a}^{\alpha, \rho} f\right)(t)\right| \leq \begin{cases}\frac{P\left(\alpha, \frac{1-\rho}{\rho}(t-a)\right)\|f\|}{(1-\rho) \alpha}, & \text { for } \rho \in(0,1), \\ \frac{(t-a)^{\alpha}\|f\|}{\Gamma(\alpha+1)}, & \text { for } \rho=1,\end{cases}
$$

for all $t \in[a, b]$.

Proof The proof follows just by following the same steps as in Lemma 3.1.

Lemma 3.3 Let $\rho \in(0,1), t_{1}, t_{2} \in[a, b]\left(t_{1} \leq t_{2}\right)$, and $\alpha>0$. Then

$$
\int_{t_{1}}^{t_{2}}(b-\tau)^{\alpha-1} e^{\frac{\rho-1}{\rho}(b-\tau)} d \tau=\frac{\rho^{\alpha} \Gamma(\alpha)}{(1-\rho)^{\alpha}}\left[P\left(\alpha, \frac{1-\rho}{\rho}\left(b-t_{1}\right)\right)-P\left(\alpha, \frac{1-\rho}{\rho}\left(b-t_{2}\right)\right)\right],
$$

where the function $P$ is defined by (22).

Proof Set $y=\frac{1-\rho}{\rho}(b-\tau)$. Then $d y=-\frac{1-\rho}{\rho} d \tau$, or $d \tau=-\frac{\rho}{1-\rho} d y$, from which it follows that

$$
\begin{aligned}
\int_{t_{1}}^{t_{2}}(b-\tau)^{\alpha-1} e^{\frac{\rho-1}{\rho}(b-\tau)} d \tau & =-\int_{\frac{1-\rho}{\rho}\left(b-t_{1}\right)}^{\frac{1-\rho}{\rho}\left(b-t_{2}\right)}\left(\frac{\rho}{1-\rho} y\right)^{\alpha-1} e^{-y} \frac{\rho}{1-\rho} d y \\
& =\frac{\rho^{\alpha}}{(1-\rho)^{\alpha}} \int_{\frac{1-\rho}{\rho}\left(b-t_{2}\right)}^{\frac{1-\rho}{\rho}\left(b-t_{1}\right)} y^{\alpha-1} e^{-y} d y
\end{aligned}
$$


Using property (26) in Lemma 2.11, we get

$$
\begin{aligned}
\int_{t_{1}}^{t_{2}}(b-\tau)^{\alpha-1} e^{\frac{\rho-1}{\rho}(b-\tau)} d \tau & =\frac{\rho^{\alpha}}{(1-\rho)^{\alpha}}\left[\gamma\left(\alpha, \frac{1-\rho}{\rho}\left(b-t_{1}\right)\right)-\gamma\left(\alpha, \frac{1-\rho}{\rho}\left(b-t_{2}\right)\right)\right] \\
& =\frac{\rho^{\alpha} \Gamma(\alpha)}{(1-\rho)^{\alpha}}\left[P\left(\alpha, \frac{1-\rho}{\rho}\left(b-t_{1}\right)\right)-P\left(\alpha, \frac{1-\rho}{\rho}\left(b-t_{2}\right)\right)\right] .
\end{aligned}
$$

The proof is completed.

Lemma 3.4 Let $\rho \in(0,1], \alpha>0$, and $a \leq \tau \leq t_{1}<t_{2} \leq b$. If either $0<\alpha \leq 1$ or $\alpha>1$, then

$$
\lim _{t_{2} \rightarrow t_{1}} \int_{a}^{t_{1}}\left|\left(t_{2}-\tau\right)^{\alpha-1} e^{\frac{\rho-1}{\rho}\left(t_{2}-\tau\right)}-\left(t_{1}-\tau\right)^{\alpha-1} e^{\frac{\rho-1}{\rho}\left(t_{1}-\tau\right)}\right| d \tau=0 .
$$

Proof To calculate the above limit, the sign to the term inside the absolute value must be studied.

For $\rho=1$, we look at the three cases $\alpha=1, \alpha<1$, and $\alpha>1$ as follows:

$$
\begin{aligned}
& \int_{a}^{t_{1}}\left|\left(t_{2}-\tau\right)^{\alpha-1} e^{\frac{\rho-1}{\rho}\left(t_{2}-\tau\right)}-\left(t_{1}-\tau\right)^{\alpha-1} e^{\frac{\rho-1}{\rho}\left(t_{1}-\tau\right)}\right|_{\rho=1} d \tau \\
& \quad=\int_{a}^{t_{1}}\left|\left(t_{2}-\tau\right)^{\alpha-1}-\left(t_{1}-\tau\right)^{\alpha-1}\right| d \tau \\
& \quad= \begin{cases}0, & \text { for } \alpha=1, \\
\frac{1}{\alpha}\left(\left(t_{2}-t_{1}\right)^{\alpha}-\left(t_{2}-a\right)^{\alpha}+\left(t_{1}-a\right)^{\alpha}\right), & \text { for } \alpha<1, \\
\frac{1}{\alpha}\left(-\left(t_{2}-t_{1}\right)^{\alpha}+\left(t_{2}-a\right)^{\alpha}-\left(t_{1}-a\right)^{\alpha}\right), & \text { for } \alpha>1,\end{cases}
\end{aligned}
$$

hence the integral has the value zero as $t_{2} \rightarrow t_{1}$.

Next, for $\rho \in(0,1)$ and $0<\alpha \leq 1$.

Because $\alpha-1 \leq 0, \frac{\rho-1}{\rho}\left(t_{2}-\tau\right) \leq 0$, and $\frac{\rho-1}{\rho}\left(t_{1}-\tau\right) \leq 0$, so we conclude that

$$
\left(t_{2}-\tau\right)^{\alpha-1} e^{\frac{\rho-1}{\rho}\left(t_{2}-\tau\right)}-\left(t_{1}-\tau\right)^{\alpha-1} e^{\frac{\rho-1}{\rho}\left(t_{1}-\tau\right)} \leq 0 .
$$

Then we get

$$
\begin{aligned}
& \int_{a}^{t_{1}}\left|\left(t_{2}-\tau\right)^{\alpha-1} e^{\frac{\rho-1}{\rho}\left(t_{2}-\tau\right)}-\left(t_{1}-\tau\right)^{\alpha-1} e^{\frac{\rho-1}{\rho}\left(t_{1}-\tau\right)}\right| d \tau \\
& \quad=\int_{a}^{t_{1}}-\left(t_{2}-\tau\right)^{\alpha-1} e^{\frac{\rho-1}{\rho}\left(t_{2}-\tau\right)} d \tau+\int_{a}^{t_{1}}\left(t_{1}-\tau\right)^{\alpha-1} e^{\frac{\rho-1}{\rho}\left(t_{1}-\tau\right)} d \tau .
\end{aligned}
$$

From Lemma 3.3, we obtain

$$
\begin{aligned}
& \int_{a}^{t_{1}}\left|\left(t_{2}-\tau\right)^{\alpha-1} e^{\frac{\rho-1}{\rho}\left(t_{2}-\tau\right)}-\left(t_{1}-\tau\right)^{\alpha-1} e^{\frac{\rho-1}{\rho}\left(t_{1}-\tau\right)}\right| d \tau \\
& \quad=\frac{\rho^{\alpha} \Gamma(\alpha)}{(1-\rho)^{\alpha}}\left\{-P\left(\alpha, \frac{1-\rho}{\rho}\left(t_{2}-a\right)\right)+P\left(\alpha, \frac{1-\rho}{\rho}\left(t_{2}-t_{1}\right)\right)\right.
\end{aligned}
$$




$$
\begin{aligned}
& \left.+P\left(\alpha, \frac{1-\rho}{\rho}\left(t_{1}-a\right)\right)-0\right\} \\
& \rightarrow 0 \text { as } t_{2} \rightarrow t_{1} .
\end{aligned}
$$

Now, for $\rho \in(0,1)$ and $\alpha>1$.

Note that $(t-\tau)^{\alpha-1} e^{\frac{\rho-1}{\rho}(t-\tau)}$ is a continuous function on $[a, b] \times[a, b]$, then it is uniformly continuous. So, for any $\epsilon>0$, there exists a constant $\delta=\delta(\epsilon)>0$ such that

$$
\left|\left(t_{2}-\tau_{2}\right)^{\alpha-1} e^{\frac{\rho-1}{\rho}\left(t_{2}-\tau_{2}\right)}-\left(t_{1}-\tau_{1}\right)^{\alpha-1} e^{\frac{\rho-1}{\rho}\left(t_{1}-\tau_{1}\right)}\right|<\epsilon
$$

for all $t_{1}, t_{2}, \tau_{1}, \tau_{2} \in[a, b]$ and $\left|t_{2}-t_{1}\right|<\delta,\left|\tau_{2}-\tau_{1}\right|<\delta$.

Then

$$
\begin{aligned}
\int_{a}^{t_{1}}\left|\left(t_{2}-\tau\right)^{\alpha-1} e^{\frac{\rho-1}{\rho}\left(t_{2}-\tau\right)}-\left(t_{1}-\tau\right)^{\alpha-1} e^{\frac{\rho-1}{\rho}\left(t_{1}-\tau\right)}\right| d \tau & \leq \epsilon \int_{a}^{t_{1}} d \tau \\
& =\left(t_{1}-a\right) \epsilon \\
& \leq(b-a) \epsilon
\end{aligned}
$$

Hence, we conclude that

$$
\int_{a}^{t_{1}}\left|\left(t_{2}-\tau\right)^{\alpha-1} e^{\frac{\rho-1}{\rho}\left(t_{2}-\tau\right)}-\left(t_{1}-\tau\right)^{\alpha-1} e^{\frac{\rho-1}{\rho}\left(t_{1}-\tau\right)}\right| d \tau \rightarrow 0 \quad \text { uniformly as } t_{2} \rightarrow t_{1} .
$$

Lemma 3.5 Let $\rho \in(0,1], \beta \geq 0$, and $h_{\beta}(t)=e^{\frac{\rho-1}{\rho}(t-a)}(t-a)^{\beta}, t \in[a, b]$. Then

$$
\max _{t \in[a, b]} h_{\beta}(t)=\bar{h}_{\beta}= \begin{cases}\left(\frac{\rho \beta}{(1-\rho) e}\right)^{\beta}, & \text { if } a+\frac{\rho \beta}{1-\rho} \in[a, b] \\ e^{\frac{\rho-1}{\rho}(b-a)}(b-a)^{\beta}, & \text { if } a+\frac{\rho \beta}{1-\rho} \notin[a, b] \text { or } \rho=1 .\end{cases}
$$

Proof It is clear that $h_{\beta}(t)$ is a continuous and nonnegative function for all $t \in[a, b]$, and $h_{\beta}(a)=0$ and $h_{\beta}(b)=e^{\frac{\rho-1}{\rho}(b-a)}(b-a)^{\beta}$. Now, differentiating the function $h_{\beta}$, we get

$$
h_{\beta}^{\prime}(t)=\left(\beta(t-a)^{\beta-1}-\frac{1-\rho}{\rho}(t-a)^{\beta}\right) e^{\frac{\rho-1}{\rho}(t-a)} .
$$

So, the equation $h_{\beta}^{\prime}(t)=0$ has a unique solution at the point

$$
t^{*}=a+\frac{\rho \beta}{1-\rho}, \quad \rho \neq 1
$$

where $h_{\beta}\left(t^{*}\right)=\left(\frac{\rho \beta}{(1-\rho) e}\right)^{\beta}$, we obtain the given result in the above lemma. The proof is completed.

\section{Some Cauchy problems in the frame of fractional proportional derivatives}

This section is devoted to applying the above proven essential lemmas to study the initial value problem (2), and then we deduce the results of problem (3). The proof of the next result follows by Theorem 5.3 in [11] or (17) in Proposition 2.9 and Lemma 2.7. 
Lemma 4.1 For $\Psi \in C([a, b], \mathbb{R})$, the solution of the following linear problem

$$
\left\{\begin{array}{l}
{ }^{c} D_{a}^{\alpha, \rho} u(t)=\Psi(t), \quad a<t<b, \alpha>0 \\
u^{(k)}(a)=b_{k}, \quad k=0,1, \ldots, n-1
\end{array}\right.
$$

is given by the integral equation

$$
u(t)=\sum_{k=0}^{n-1} \frac{\varphi_{k}}{\rho^{k} k !}(t-a)^{k} e^{\frac{\rho-1}{\rho}(t-a)}+\frac{1}{\rho^{\alpha} \Gamma(\alpha)} \int_{a}^{t}(t-\tau)^{\alpha-1} e^{\frac{\rho-1}{\rho}(t-\tau)} \Psi(\tau) d \tau,
$$

where $\varphi_{k}=\sum_{j=0}^{k}\left(\begin{array}{l}k \\ j\end{array}\right) \rho^{j}(1-\rho)^{k-j} b_{k}$ for $\rho \in(0,1)$ and $\varphi_{k}=b_{k}$ for $\rho=1$.

Let $X=C([a, b], \mathbb{R})$ be a Banach space of all continuous functions from $[a, b]$ to $\mathbb{R}$ endowed with the norm $\|u\|=\sup _{t \in[a, b]}|u(t)|$.

Associated with problem (2), we define a fixed point operator $T: X \rightarrow X$ by

$$
T u(t)=\sum_{k=0}^{n-1} \frac{\varphi_{k}}{\rho^{k} k !}(t-a)^{k} e^{\frac{\rho-1}{\rho}(t-a)}+\frac{1}{\rho^{\alpha} \Gamma(\alpha)} \int_{a}^{t}(t-\tau)^{\alpha-1} e^{\frac{\rho-1}{\rho}(t-\tau)} f(\tau, u(\tau)) d \tau .
$$

Consider the following hypothesis:

$\left(H_{1}\right) f:[a, b] \times \mathbb{R} \rightarrow \mathbb{R}$ is a continuous function and there exists $k>0$ such that $f(t, A)$ $f(t, B)|\leq L| A-B \mid$ for all $t \in[a, b], A, B \in \mathbb{R}$, and $|f(t, 0)| \leq \Omega(t)$, with $\Omega$ being a continuous and nonnegative function where $\bar{\Omega}=\sup _{t \in[a, b]} \Omega(t)$.

Theorem 4.2 Let $\rho \in(0,1)$, and assume that $\left(H_{1}\right)$ holds. If either $0<\alpha \leq 1$ or $\alpha>1$, then problem (2) has a unique solution on $[a, b]$ if

$$
\frac{L P\left(\alpha, \frac{1-\rho}{\rho}(b-a)\right)}{(1-\rho)^{\alpha}}<1
$$

where $P$ is defined by means of (22).

Proof Let us choose $R>0$ satisfying

$$
R \geq \frac{\sum_{k=0}^{n-1} \frac{\left|\varphi_{k}\right| \bar{h}_{k}}{\rho^{k} k !}+\frac{\bar{\Omega}}{(1-\rho)^{\alpha}} P\left(\alpha, \frac{1-\rho}{\rho}(b-a)\right)}{1-\frac{L}{(1-\rho)^{\alpha}} P\left(\alpha, \frac{1-\rho}{\rho}(b-a)\right)},
$$

where $\bar{h}_{k}$ is defined in Lemma 3.5 with $k \in\{0,1, \ldots, n-1\}$, and consider $M_{R}=\{u \in X$ : $\|u\| \leq R\}$. We first show that $T M_{R} \subset M_{R}$.

For $u \in M_{R}$ and $t \in[a, b]$, we have

$$
\begin{aligned}
|T u(t)| \leq & \sum_{k=0}^{n-1} \frac{\left|\varphi_{k}\right|}{\rho^{k} k !}(t-a)^{k} e^{\frac{\rho-1}{\rho}(t-a)}+\frac{1}{\rho^{\alpha} \Gamma(\alpha)} \int_{a}^{t}(t-\tau)^{\alpha-1} e^{\frac{\rho-1}{\rho}(t-\tau)} \\
& \times|f(\tau, u(\tau))-f(\tau, 0)+f(\tau, 0)| d \tau \\
\leq & \sum_{k=0}^{n-1} \frac{\left|\varphi_{k}\right|}{\rho^{k} k !}(t-a)^{k} e^{\frac{\rho-1}{\rho}(t-a)}+\frac{L R+\bar{\Omega}}{\rho^{\alpha} \Gamma(\alpha)} \int_{a}^{t}(t-\tau)^{\alpha-1} e^{\frac{\rho-1}{\rho}(t-\tau)} d \tau .
\end{aligned}
$$


Using Lemma 3.5 and Lemma 3.2, we obtain

$$
\begin{aligned}
|T u(t)| & \leq \sum_{k=0}^{n-1} \frac{\left|\varphi_{k}\right| \bar{h}_{k}}{\rho^{k} k !}+\frac{L R+\bar{\Omega}}{\rho^{\alpha} \Gamma(\alpha)} P\left(\alpha, \frac{1-\rho}{\rho}(b-a)\right) \\
& \leq R
\end{aligned}
$$

which implies that $\|T u\| \leq R$ for any $u \in M_{R}$. We get $T M_{R} \subseteq M_{R}$.

Next we prove that the operator $T$ is a contraction mapping. For $u, v \in X$, for all $t \in[a, b]$, we have

$$
\begin{aligned}
|T u(t)-T v(t)| & \leq \frac{1}{\rho^{\alpha} \Gamma(\alpha)} \int_{a}^{t}(t-\tau)^{\alpha-1} e^{\frac{\rho-1}{\rho}(t-\tau)}|f(\tau, u(\tau))-f(\tau, v(\tau))| d \tau \\
& \leq \frac{L\|u-v\|}{\rho^{\alpha} \Gamma(\alpha)} \int_{a}^{t}(t-\tau)^{\alpha-1} e^{\frac{\rho-1}{\rho}(t-\tau)} d \tau \\
& \leq \frac{L P\left(\alpha, \frac{1-\rho}{\rho}(b-a)\right)}{(1-\rho)^{\alpha}}\|u-v\| .
\end{aligned}
$$

Taking the supremum over all $t \in[a, b]$ yields

$$
\|T u-T v\| \leq \frac{L P\left(\alpha, \frac{1-\rho}{\rho}(b-a)\right)}{(1-\rho)^{\alpha}}\|u-v\| .
$$

By condition (41) the operator $T$ is a contraction. Hence, by the Banach fixed point theorem, problem (2) has a unique solution on $[a, b]$. The proof is completed.

Now, based on Leray-Schauder alternative fixed point theorem [30], we present the following result about the existence of solutions for the investigated problem (2).

Consider the following hypothesis:

$\left(H_{2}\right) f:[a, b] \times \mathbb{R} \rightarrow \mathbb{R}$ is a continuous functions, and there exist real positive constants $\varsigma_{0}, \varsigma_{1}$ such that

$$
|f(t, u)| \leq \varsigma_{0}+\varsigma_{1}|u|
$$

for all $(t, u) \in[a, b] \times \mathbb{R}$.

Theorem 4.3 Let $\rho \in(0,1)$, and assume that $\left(H_{2}\right)$ holds. If

$$
\frac{\varsigma_{1} P\left(\alpha, \frac{1-\rho}{\rho}(b-a)\right)}{(1-\rho)^{\alpha}}<1
$$

then the initial value problem (2) has at least one solution on $[a, b]$.

Proof We first show that the operator $T$ is completely continuous.

The continuity of $f$ implies the continuity of the operator $T$. Let $\Upsilon$ be any nonempty bounded subset of $X$. Then there exists $\xi>0$ such that, for any $u \in \Upsilon,\|u\| \leq \xi$. Notice that from condition $\left(\mathrm{H}_{2}\right)$, for all $u \in \Upsilon$, we have

$$
|f(t, u(t))| \leq \varsigma_{0}+\varsigma_{1} \xi
$$


Next we prove that $T(\Upsilon)$ is uniformly bounded. Let $u \in \Upsilon$. Then, for any $t \in[a, b]$, we have

$$
\begin{aligned}
& |\operatorname{Tu}(t)| \\
& \quad \leq \sum_{k=0}^{n-1} \frac{\left|\varphi_{k}\right|}{\rho^{k} k !}(t-a)^{k} e^{\frac{\rho-1}{\rho}(t-a)}+\frac{1}{\rho^{\alpha} \Gamma(\alpha)} \int_{a}^{t}(t-\tau)^{\alpha-1} e^{\frac{\rho-1}{\rho}(t-\tau)}|f(\tau, u(\tau))| d \tau \\
& \quad \leq \sum_{k=0}^{n-1} \frac{\left|\varphi_{k}\right|}{\rho^{k} k !}(t-a)^{k} e^{\frac{\rho-1}{\rho}(t-a)}+\frac{\varsigma_{0}+\varsigma_{1} \xi}{\rho^{\alpha} \Gamma(\alpha)} \int_{a}^{t}(t-\tau)^{\alpha-1} e^{\frac{\rho-1}{\rho}(t-\tau)} d \tau \\
& \quad \leq \sum_{k=0}^{n-1} \frac{\left|\varphi_{k}\right| \bar{h}_{k}}{\rho^{k} k !}+\frac{\zeta_{0}+\varsigma_{1} \xi}{(1-\rho)^{\alpha}} P\left(\alpha, \frac{1-\rho}{\rho}(b-a)\right) \\
& \quad<+\infty .
\end{aligned}
$$

Consequently, $\|u\|<+\infty$ for any $u \in \Upsilon$. Therefore, $T(\Upsilon)$ is uniformly bounded.

Now we show that $T$ is equicontinuous on $\Upsilon$. Let $u \in \Upsilon$. For any $t_{1}, t_{2} \in[a, b]$, where $t_{2}>t_{1}$, we have

$$
\begin{aligned}
&\left|T u\left(t_{2}\right)-T u\left(t_{1}\right)\right| \\
&=\mid \sum_{k=0}^{n-1} \frac{\varphi_{k}}{\rho^{k} k !}\left(\left(t_{2}-a\right)^{k} e^{\frac{\rho-1}{\rho}\left(t_{2}-a\right)}-\left(t_{1}-a\right)^{k} e^{\frac{\rho-1}{\rho}\left(t_{1}-a\right)}\right) \\
& \quad+\frac{1}{\rho^{\alpha} \Gamma(\alpha)} \int_{a}^{t_{2}}\left(t_{2}-\tau\right)^{\alpha-1} e^{\frac{\rho-1}{\rho}\left(t_{2}-\tau\right)}|f(\tau, u(\tau))| d \tau \\
&-\frac{1}{\rho^{\alpha} \Gamma(\alpha)} \int_{a}^{t_{1}}\left(t_{1}-\tau\right)^{\alpha-1} e^{\frac{\rho-1}{\rho}\left(t_{1}-\tau\right)}|f(\tau, u(\tau))| d \tau \mid \\
&= \mid \sum_{k=0}^{n-1} \frac{\varphi_{k}}{\rho^{k} k !}\left(\left(t_{2}-a\right)^{k} e^{\frac{\rho-1}{\rho}\left(t_{2}-a\right)}-\left(t_{1}-a\right)^{k} e^{\frac{\rho-1}{\rho}\left(t_{1}-a\right)}\right) \\
&+\frac{1}{\rho^{\alpha} \Gamma(\alpha)} \int_{a}^{t_{1}}\left(\left(t_{2}-\tau\right)^{\alpha-1} e^{\frac{\rho-1}{\rho}\left(t_{2}-\tau\right)}-\left(t_{1}-\tau\right)^{\alpha-1} e^{\frac{\rho-1}{\rho}\left(t_{1}-\tau\right)}\right)|f(\tau, u(\tau))| d \tau \\
&+\frac{1}{\rho^{\alpha} \Gamma(\alpha)} \int_{t_{2}}^{t_{1}}\left(\left(t_{2}-\tau\right)^{\alpha-1} e^{\frac{\rho-1}{\rho}\left(t_{2}-\tau\right)}\right)|f(\tau, u(\tau))| d \tau \mid .
\end{aligned}
$$

From Lemma 3.3, we have

$$
\begin{aligned}
& \left|T u\left(t_{2}\right)-T u\left(t_{1}\right)\right| \\
& \leq \sum_{k=0}^{n-1} \frac{\left|\varphi_{k}\right|}{\rho^{k} k !}\left|\left(t_{2}-a\right)^{k} e^{\frac{\rho-1}{\rho}\left(t_{2}-a\right)}-\left(t_{1}-a\right)^{k} e^{\frac{\rho-1}{\rho}\left(t_{1}-a\right)}\right| \\
& \quad+\frac{\zeta_{0}+\zeta_{1} \xi}{\rho^{\alpha} \Gamma(\alpha)} \int_{a}^{t_{1}}\left|\left(t_{2}-\tau\right)^{\alpha-1} e^{\frac{\rho-1}{\rho}\left(t_{2}-\tau\right)}-\left(t_{1}-\tau\right)^{\alpha-1} e^{\frac{\rho-1}{\rho}\left(t_{1}-\tau\right)}\right| d \tau \\
& \quad+\frac{\zeta_{0}+\varsigma_{1} \xi}{(1-\rho)^{\alpha}}\left[0-P\left(\alpha, \frac{1-\rho}{\rho}\left(t_{2}-t_{1}\right)\right)\right] .
\end{aligned}
$$


Because $h_{k}(t)=(t-a)^{k} e^{\frac{\rho-1}{\rho}(t-a)}, k=0, \ldots, n-1$, are continuous functions, then $\mid h_{k}\left(t_{2}\right)-$ $h_{k}\left(t_{1}\right) \mid \rightarrow 0$ as $t_{2} \rightarrow t_{1}$.

Then, by using Lemma 3.4, we obtain

$$
\lim _{t_{2} \rightarrow t_{1}}\left|T u\left(t_{2}\right)-T u\left(t_{1}\right)\right|=0
$$

Thus, the operator $T$ is equicontinuous. Hence, by Arzela-Ascoli theorem, we deduce that the operator $T$ is completely continuous.

Finally, we will verify that the set $M(T)=\{u \in X: u=m T u$ for some $0<m<1\}$ is bounded.

For all $u \in M(T)$, and for any $t \in[a, b]$, we have

$$
\begin{aligned}
|u(t)| & =m|T u(t)| \\
& \leq \sum_{k=0}^{n-1} \frac{\left|\varphi_{k}\right|}{\rho^{k} k !}(t-a)^{k} e^{\frac{\rho-1}{\rho}(t-a)}+\frac{1}{\rho^{\alpha} \Gamma(\alpha)} \int_{a}^{t}(t-\tau)^{\alpha-1} e^{\frac{\rho-1}{\rho}(t-\tau)}|f(\tau, u(\tau))| d \tau \\
& \leq \sum_{k=0}^{n-1} \frac{\left|\varphi_{k}\right|}{\rho^{k} k !}(t-a)^{k} e^{\frac{\rho-1}{\rho}(t-a)}+\frac{\varsigma_{0}+\varsigma_{1}\|u\|}{\rho^{\alpha} \Gamma(\alpha)} \int_{a}^{t}(t-\tau)^{\alpha-1} e^{\frac{\rho-1}{\rho}(t-\tau)} d \tau \\
& \leq \sum_{k=0}^{n-1} \frac{\left|\varphi_{k}\right| \bar{h}_{k}}{\rho^{k} k !}+\frac{\varsigma_{0}}{(1-\rho)^{\alpha}} P\left(\alpha, \frac{1-\rho}{\rho}(b-a)\right)+\frac{\varsigma_{1}\|u\|}{(1-\rho)^{\alpha}} P\left(\alpha, \frac{1-\rho}{\rho}(b-a)\right),
\end{aligned}
$$

which yields

$$
\|u\| \leq \frac{\sum_{k=0}^{n-1} \frac{\left|\hat{\varphi}_{k}\right| \bar{h}_{k}}{\rho^{k} k !}+\frac{\varsigma 0}{(1-\rho)^{\alpha}} P\left(\alpha, \frac{1-\rho}{\rho}(b-a)\right)}{1-\frac{\varsigma 1}{(1-\rho)^{\alpha}} P\left(\alpha, \frac{1-\rho}{\rho}(b-a)\right)},
$$

which proves that $M$ is bounded. Thus, by Leray-Schauder alternative theorem, the operator $T$ has at least one fixed point. Hence, the initial value problem (2) has at least one solution on $[a, b]$. The proof is completed.

Remark 4.4 From Lemma (3.1), in the case $\rho=1$, we can replace the formula $\frac{P\left(\alpha, \frac{1-\rho}{\rho}(b-a)\right)}{(1-\rho)^{\alpha}}$ by the formula $\frac{(b-a)^{\alpha}}{\Gamma(\alpha+1)}$ So, then we can conclude the usual results for the existence and uniqueness of the solution of the Cauchy problem with usual Caputo fractional derivative.

Because $P(\alpha, x) \in[0,1]$ for all $\alpha, x \in \mathbb{R}^{+}$, we obtain the following results.

Corollary 4.5 Let $\rho \in(0,1)$, and assume that $\left(H_{1}\right)$ holds. Then problem (2) has a unique solution on $[a, b]$ if

$$
\frac{L}{(1-\rho)^{\alpha}}<1
$$

Corollary 4.6 Let $\rho \in(0,1)$, and assume that $\left(H_{2}\right)$ holds. If

$$
\frac{\varsigma_{1}}{(1-\rho)^{\alpha}}<1
$$

then the initial value problem (2) has at least one solution on $[a, b]$. 
Now, concerning the study of the initial value problem of Riemann-Liouville type (3), we present the following results.

Lemma 4.7 For $\Psi \in C([a, b], \mathbb{R})$, the solution of the following linear problem

$$
\left\{\begin{array}{l}
{ }^{R} D_{a}^{\alpha, \rho} u(t)=\Psi(t), \quad a<t<b, \alpha>0, \\
{ }^{R} D_{a}^{\alpha-k} u(a)=b_{k}, \quad k=1, \ldots, n,
\end{array}\right.
$$

is given by

$$
\begin{aligned}
u(t)= & \sum_{k=1}^{n} \frac{b_{k}}{\rho^{\alpha-k} \Gamma(\alpha-k+1)}(t-a)^{\alpha-k} e^{\frac{\rho-1}{\rho}(t-a)} \\
& +\frac{1}{\rho^{\alpha} \Gamma(\alpha)} \int_{a}^{t}(t-\tau)^{\alpha-1} e^{\frac{\rho-1}{\rho}(t-\tau)} \Psi(\tau) d \tau .
\end{aligned}
$$

Proof Applying the operator $J_{a^{+}}^{\alpha, \rho}$ on equation (47), with using (18), we get

$$
u(t)=\sum_{k=1}^{n} q_{k}(t-a)^{\alpha-k} e^{\frac{\rho-1}{\rho}(t-a)}+J_{a}^{\alpha, \rho} \Psi(t),
$$

where $q_{k} \in \mathbb{R}, k \in\{1,2, \ldots, n\}$.

Now, applying the operator ${ }^{R} D_{a}^{\alpha-k, \rho}$ on (49), we get

$$
\begin{aligned}
{ }^{R} D_{a}^{\alpha-k, \rho} u(t)= & \sum_{i=1}^{n} q_{j}^{R} D_{a}^{\alpha-k, \rho}\left((t-a)^{\alpha-i} e^{\frac{\rho-1}{\rho}(t-a)}\right)+{ }^{R} D_{a}^{\alpha-k, \rho} J_{a}^{\alpha, \rho} \Psi(t) \\
= & \sum_{i=1}^{n} q_{i} e^{\frac{1-\rho}{\rho} a R} D_{a}^{\alpha-k, \rho}\left((t-a)^{\alpha-i} e^{\frac{\rho-1}{\rho} t}\right)+J_{a}^{k, \rho} \Psi(t) \\
= & \sum_{i=1}^{n} q_{i} \frac{\rho^{\alpha-k} \Gamma(\alpha-i+1)}{\Gamma(k-i+1)}(t-a)^{k-i} e^{\frac{\rho-1}{\rho}(t-a)}+J_{a}^{k, \rho} \Psi(t) \\
= & \sum_{\substack{i=1 \\
i \neq k}}^{n} q_{i} \frac{\rho^{\alpha-k} \Gamma(\alpha-i+1)}{\Gamma(k-i+1)}(t-a)^{k-i} e^{\frac{\rho-1}{\rho}(t-a)} \\
& +q_{k} \rho^{\alpha-k} \Gamma(\alpha-k+1) e^{\frac{\rho-1}{\rho}(t-a)}+J_{a}^{k, \rho} \Psi(t) .
\end{aligned}
$$

So, for $t=a$, we obtain

$$
{ }^{R} D_{a}^{\alpha-k, \rho} u(a)=q_{k} \rho^{\alpha-k} \Gamma(\alpha-k+1) .
$$

For any $k \in\{1,2, \ldots, n\}$, using the initial condition $\left({ }^{R} D_{a}^{\alpha-k, \rho} u\right)(a)=b_{k}$, we get $q_{k}=$ $\frac{b_{k}}{\rho^{\alpha-k} \Gamma(\alpha-k+1)}$.

Substituting the values $q_{k}(k \in\{1,2, \ldots, n\})$ in (49), we obtain the integral equation (47). The proof is completed. 
Associated with problem (3), we define a fixed point operator $\widetilde{T}: X \rightarrow X$ by

$$
\begin{aligned}
\widetilde{T} u(t)= & \sum_{k=1}^{n} \frac{b_{k}(t-a)^{\alpha-k}}{\rho^{\alpha-k} \Gamma(\alpha-k+1)} e^{\frac{\rho-1}{\rho}(t-a)} \\
& +\frac{1}{\rho^{\alpha} \Gamma(\alpha)} \int_{a}^{t}(t-\tau)^{\alpha-1} e^{\frac{\rho-1}{\rho}(t-\tau)} f(\tau, u(\tau)) d \tau .
\end{aligned}
$$

Remark 4.8 It is noticeable that the two operators $T$ and $\widetilde{T}$ are similar in form. So, in the same way as the above study of the proportional fractional problem of Caputo type (3), the reader can easily check that the above results we came up with (Theorem 4.2, Theorem 4.3, Corollary 4.5, and Corollary 4.6) can be also applied with the same conditions on the Riemann-Liouville proportional fractional problem (3).

Example 4.9 Consider the following initial values problem with GPF of Caputo type:

$$
\left\{\begin{array}{l}
{ }^{c} D_{0}^{3 / 2,1 / 2} u(t)=(t-1)^{2}+\frac{1}{2} \sin u(t), \quad 0<t \leq 1, \\
u(0)=A_{1}, \quad u^{\prime}(0)=A_{2}, \quad A_{1}, A_{2} \in \mathbb{R} .
\end{array}\right.
$$

Here, $\alpha=\frac{3}{2}, \rho=\frac{1}{2}, a=0, b=1$ and $f(t, u)=(t-1)^{2}+\sin u$. For all $(t, u) \in[0,1] \times \mathbb{R}$, we have

$$
\begin{aligned}
\left|\partial_{u} f(t, u)\right| & =\frac{1}{2}|\cos u| \\
& \leq \frac{1}{2}:=L .
\end{aligned}
$$

Using Matlab program with the given values, we obtain

$$
\frac{L P\left(\alpha, \frac{1-\rho}{\rho}(b-a)\right)}{(1-\rho)^{\alpha}}=0.604708<1 .
$$

Then inequality (41) is satisfied. Hence, by Theorem 4.2, we conclude that the GPF problem (52) has a unique solution on the interval $[0,1]$.

Example 4.10 Consider the following initial values problem with GPF of RiemannLiouville type:

$$
\left\{\begin{array}{l}
{ }^{R} D_{0}^{3 / 2,1 / 2} u(t)=1-t+\frac{3}{4} \ln (1+|u(t)|), \quad 0<t \leq 1, \\
u(0)=A_{1}, \quad u^{\prime}(0)=A_{2}, \quad A_{1}, A_{2} \in \mathbb{R} .
\end{array}\right.
$$

Here, $\alpha=\frac{3}{2}, \rho=\frac{1}{2}, a=0, b=1$ and $f(t, u)=1-t+\frac{3}{4} \ln (1+|u|)$.

For all $(t, u) \in[0,1] \times \mathbb{R}$, we have

$$
|f(t, u)| \leq \frac{7}{4}+\frac{3}{4}|u|
$$

choose $\varsigma_{1}=3 / 4$. 
Using Matlab program with the given values, we obtain

$$
\frac{\zeta_{1} P\left(\alpha, \frac{1-\rho}{\rho}(b-a)\right)}{(1-\rho)^{\alpha}}=0.907062<1 .
$$

Then inequality (43) is satisfied. Hence, by Remark 4.8 and Theorem 4.3, we conclude that the GPF problem (53) has at least one solution on $[0,1]$.

\section{Acknowledgements}

The authors would like to thank the editor and reviewers for their valuable comments which improved the manuscript.

Funding

Not applicable.

Availability of data and materials

Data sharing not applicable to this article as no data sets were generated or analysed during the current study.

\section{Competing interests}

The authors declare that they have no competing interests.

\section{Authors' contributions}

All authors equally contributed in this manuscript and approved the final version.

\section{Author details}

'Department of Mathematics and Computer Sciences, ICOSI Laboratory, Abbes Laghrour University, Khenchela, 40004, Algeria. ${ }^{2}$ Department of Mathematics and General Sciences, Prince Sultan University, P.O. Box 66833, 11586 Riyadh, Saudi Arabia. ${ }^{3}$ Department of Medical Research, China Medical University, 40402 Taichung, Taiwan. ${ }^{4}$ Department of Computer Science and Information Engineering, Asia University, Taichung, Taiwan. ${ }^{5}$ Department of Mathematics, Çankaya University, 06790, Etimesgut Ankara, Turkey.

\section{Publisher's Note}

Springer Nature remains neutral with regard to jurisdictional claims in published maps and institutional affiliations.

Received: 16 June 2020 Accepted: 8 October 2020 Published online: 16 November 2020

\section{References}

1. Podlubny, I.: Fractional Differential Equations. Academic Press, San Diego (1999)

2. Samko, S.G., Kilbas, A.A., Marichev, O.I.: Fractional Integrals and Derivatives: Theory and Applications. Gordon \& Breach, Yverdon (1993)

3. Hilfer, R.: Applications of Fractional Calculus in Physics. Word Scientific, Singapore (2000)

4. Debnath, L.: Recent applications of fractional calculus to science and engineering. Int. J. Math. Math. Sci. $\mathbf{5 4}$ 3413-3442 (2003)

5. Kilbas, A., Srivastava, H.M., Trujillo, J...: Theory and Application of Fractional Differential Equations. North Holland Mathematics Studies, vol. 204. North-Holland, Amsterdam (2006)

6. Magin, R.L.: Fractional Calculus in Bioengineering. Begell House Publishers (2006)

7. Khalil, R., Al Horani, M., Yousef, A., Sababheh, M.: A new definition of fractional derivative. J. Comput. Appl. Math. 264, 65-70 (2014)

8. Abdeljawad, T.: On conformable fractional calculus. J. Comput. Appl. Math. 279, 57-66 (2013)

9. Anderson, D.R., Ulness, D.J.: Newly defined conformable derivatives. Adv. Dyn. Syst. Appl. 10(2), 109-137 (2015)

10. Jarad, F., Uğurlu, E., Abdeljawad, T., Baleanu, D.: On a new class of fractional operators. Adv. Differ. Equ. 2018, 142 (2018)

11. Jarad, F., Abdeljawad, T., Alzabut, J.: Generalized fractional derivatives generated by a class of local proportional derivatives. Eur. Phys. J. Spec. Top. 226, 3457-3471 (2017)

12. Katugampola, U.N. New approach to generalized fractional integral. Appl. Math. Comput. 218, 860-865 (2011)

13. Katugampola, U.N.: A new approach to generalized fractional derivatives. Bull. Math. Anal. Appl. 6(4), 1-15 (2014)

14. Jarad, F., Abdeljawad, T.: Generalized fractional derivatives and Laplace transform. Discrete Contin. Dyn. Syst. 13(3), 709-722 (2020)

15. Rashid, S., Jarad, F., Noor, M.A., Kalsoom, H., Chu, Y.: Inequalities by means of generalized proportional fractional integral operators with respect to another function. Mathematics 7, no. 12 (2019). https://doi.org/10.3390/math7121225

16. Jarad, F., Alqudah, M.A., Abdeljawad, T.: On more general forms of proportional fractional operators. Open Math. 18 167-176 (2020)

17. Baleanu, D., Fernandez, A., Akkgul, A.: On a fractional operator combining proportional and classical differintegrals. Mathematics 8, Article Number 360 (2020). https://doi.org/10.3390/math8030360

18. Caputo, M., Fabrizio, M.: A new definition of fractional derivative without singular kernel. Prog. Fract. Differ. Appl. 1, 73-85 (2015) 
19. Atangana, A., Baleanu, D.: New fractional derivative with non-local and non-singular kernel. Therm. Sci. 20, 757-763 (2016)

20. Abdeljawad, T.: Fractional operators with generalized Mittag-Leffler kernels and their iterated differintegrals. Chaos 29, 023102 (2019). https://doi.org/10.1063/1.5085726

21. Ahmad, B., Nieto, J.J.: Existence results for nonlinear boundary value problems of fractional integrodifferential equations with integral boundary conditions. Bound. Value Probl. 2009, 708576 (2009)

22. Wang, G., Ghanmi, A., Horrigue, S., Madian, S.: Existence result and uniqueness for some fractional problem. Mathematics 7, 516 (2019)

23. Ali, K.B., Ghanmi, A., Kefi, K.: Existence of solutions for fractional differential equations with Dirichlet boundary conditions. Electron. J. Differ. Equ. 2016, 116, 1-11 (2016)

24. Zhang, W., Liu, W., Xue, T.: Existence and uniqueness results for the coupled systems of implicit fractional differential equations with periodic boundary conditions. Adv. Differ. Equ. 2018, 413 (2018)

25. Borisut, P., Kumam, P., Ahmad, I., Sitthithakerngkiet, K.: Nonlinear Caputo fractional derivative with nonlocal Riemann-Liouville fractional integral condition via fixed point theorems. Symmetry 11(6), 829 (2019)

26. Shaoib, M., Abdeljawad, T., Sarwar, M., Jarad, F.: Fixed point theorems for multi-valued contractions in metric spaces with applications to fractional differential and integral equations. IEEE Access 7, 127373-127383 (2019)

27. Alqahtani, B., Fulga, A., Jarad, F., Karapinar, E.: Nonlinear F-contractions on b-metric spaces and differential equations in the frame of fractional derivatives with Mittag-Leffler kernel. Chaos Solitons Fractals 128, 349-354 (2019)

28. Erdélyi, A., Magnus, W., Oberhettinger, F., Tricomi, F.G.: Higher Transcendental Functions, Vol. II. McGraw-Hill, New York (1953)

29. Gil, A., Segura, J., Temme, N.M.: Efficient and accurate algorithms for the computation and inversion of the incomplete gamma function ratios. SIAM J. Sci. Comput. 34(6), A2965-A2981 (2012)

30. Granas, A., Dugundji, J.: Fixed Point Theory. Springer, New York (2003)

\section{Submit your manuscript to a SpringerOpen ${ }^{\circ}$ journal and benefit from:}

- Convenient online submission

- Rigorous peer review

- Open access: articles freely available online

- High visibility within the field

- Retaining the copyright to your article

Submit your next manuscript at $\boldsymbol{\nabla}$ springeropen.com 